\title{
Examining the effect of practice on inhibition of return in static displays
}

\author{
JAY PRATT \\ University of Toronto, Toronto, Ontario, Canada \\ and \\ JIM MCAULFFE \\ Lakehead University, Thunder Bay, Ontario, Canada
}

\begin{abstract}
In a recent article, Weaver, Lupiáñez, and Watson (1998) reported that both object-based and location-based inhibition of return effects were reduced with practice. The present study was conducted to (1) replicate the reduction of inhibition of return with practice in single-session experiments with a variety of displays and responses and (2) to examine the notion that the reduction was, at least partly, due to habituation. However, no evidence for practice-related changes in the size of the inhibitory effect were found over a series of different inhibition of return experiments using static displays (using various numbers of target locations, types of keypress responses, and number of trials). Overall, the results suggest that inhibition of return is a robust phenomenon and may not, with static displays, be especially sensitive to practice effects.
\end{abstract}

It is a well-documented finding that practice affects performance in a wide variety of tasks. Generally, we are able to perform tasks faster and more accurately when we have had practice. In addition to reductions in reaction time (RT) (Mowbray \& Rhoades, 1959), movement time (Pratt \& Abrams, 1996), and error rates (Proteau, Blandin, Alain, \& Dorian, 1994) with practice, other systematic changes may occur. For example, LaBerge and Samuels (1974) found that with over 5 days of practice, people were able to acquire a more automatic manner of information processing. Recently, Weaver, Lupiáñez, and Watson (1998) presented evidence that practice may affect a phenomenon known as inhibition of return.

A typical inhibition of return paradigm involves a display consisting of two peripheral target locations separated by a central fixation point. Following the presentation of a cue (such as an abrupt onset) at one of the peripheral locations, there is a brief period of time in which targets that occur at the cued location are detected faster than targets that occur at uncued (or novel) locations. However, after this short period of facilitation (which typically lasts approximately $300 \mathrm{msec}$ after the onset of the cue), there occurs a much longer period of time in which targets at the cued location are detected more slowly than are targets that occur at uncued locations. This inhibitory period

This research was supported by National Science and Engineering Council of Canada awards (0194537 and 0196162) to J.P. We thank Jeremy Wolfe, Todd Horowitz, Tram Neill, and Thomas Sanocki for their comments on an earlier version of this manuscript. We also thank Avdeep Marwa for her assistance in the data collection. Correspondence should be addressed to J. Pratt, Department of Psychology, $100 \mathrm{St}$. George Street, University of Toronto, Toronto, ON M5S 3G3, Canada (e-mail: pratt@psych.utoronto.ca). typically lasts anywhere from 300 to $2,000 \mathrm{msec}$ after the onset of the cue and has become known as inhibition of return. The term reflects the notion that the inhibition may be due to an attentional mechanism that inhibits attention from returning to previously attended locations (Posner \& Cohen, 1984). The utility of such a mechanism may be to make visual and/or attentional searches more efficient by biasing attention toward novel locations and away from locations that have already been examined (see, e.g., Tipper, Weaver, Jerreat, \& Burak, 1994). It should be noted, however, that although Klein (1988) found evidence of inhibition of return in a serial, but not a parallel, visual search task (as might be expected if inhibition of return is attentional in nature), Wolfe and Pokorny (1990) failed to replicate this result, finding inhibition in both search tasks (as did Klein in a later experiment, reported in Klein \& Taylor, 1994). Thus, although considerable evidence suggests that there is a large attentional component to inhibition of return, the exact nature of the inhibition has yet to be determined.

Since the original finding of inhibition of return by Posner and Cohen (1984), the effect has proven to be very robust. Inhibition of return has been found in detection tasks with simple keypress responses (e.g., Maylor \& Hockey, 1985), choice keypress responses (e.g., Maylor, 1985), and eye movement responses (e.g., Abrams \& Dobkin, 1994). Recently, inhibition of return has also been found in discrimination tasks in which the responses were based on either the identity of the target stimulus (Lupiáñez, Milán, Tornay, Madrid, \& Tudela, 1997; Pratt, Kingstone, \& Khoe, 1997) or the identity and location of the target stimulus (Pratt, 1995; Pratt \& Abrams, 1999; but see also Terry, Valdes, \& Neill, 1994). An especially interesting finding has been that the inhibition appears not 
only to occur at the cued location (location-based inhibition of return) but also to stay with a cued object even when that object moves away from the location where it was cued (e.g., Abrams \& Dobkin, 1994; Tipper, Driver, $\&$ Weaver, 1991; Tipper et al., 1994). This effect has come to be known as object-based inhibition of return (e.g., Tipper et al., 1991). However, in an seven-experiment study, Müller and von Mühlenen (1996) failed to find any evidence for object-based inhibition of return and instead found evidence that cued objects may yield facilitated, not inhibited, detection responses under some circumstances (if they occur in the upper visual field and move from left to right).

In trying to reconcile the disparate results regarding object-based inhibition of return, Weaver et al. (1998) noted that participants in the Müller and von Mühlenen (1996) study were given extensive practice sessions before the experimental session in which data were collected. They suggested that inhibition of return may decrease with practice due to a combination of effects and that Müller and von Mühlenen did not find inhibition of return in their experimental sessions because of these practice-related effects. One of the effects Weaver et al. suggested for the decrease in inhibition was habituation, a decrease in responsivity that often occurs with repeated presentations of a stimulus. The other effect suggested by Weaver et al. has to do with the notion that cuing an object may result in both facilitatory and inhibitory pro- cessing and that the inhibitory component may decrease with practice, whereas the facilitatory component does not (or, at least, decreases at a slower rate with practice).

To test their notion that inhibition of return decreases with practice, Weaver et al. (1998) conducted two experiments in which the magnitude of the inhibition effect was examined over the course of 1,080 test trials and 720 catch trials per participant (collected in four blocks of trials on 3 successive days). The first experiment examined object-based inhibition and location-based inhibition. Following the cue in one of the three peripheral target boxes, the boxes were rotated $120^{\circ}$. The target could then occur at the cued object (which had moved to a new location), the cued location (to which a new object had moved), or at the uncued box (which was neither the cued object nor the cued location). The second experiment, in which the objects remained static after the presentation of the cue, examined the additive effect of object-based and location-based inhibition (i.e., the combination of inhibition to the cued object and inhibition to the cued location). Their results indicated that inhibition of return decreased with practice for the object-based component (first experiment), the location-based component (first experiment), and the combination of the two components (second experiment).

Overall, the decrease in inhibition of return reported by Weaver et al. (1998) was quite marked. In the first experiment, the magnitude of the object-based inhibition

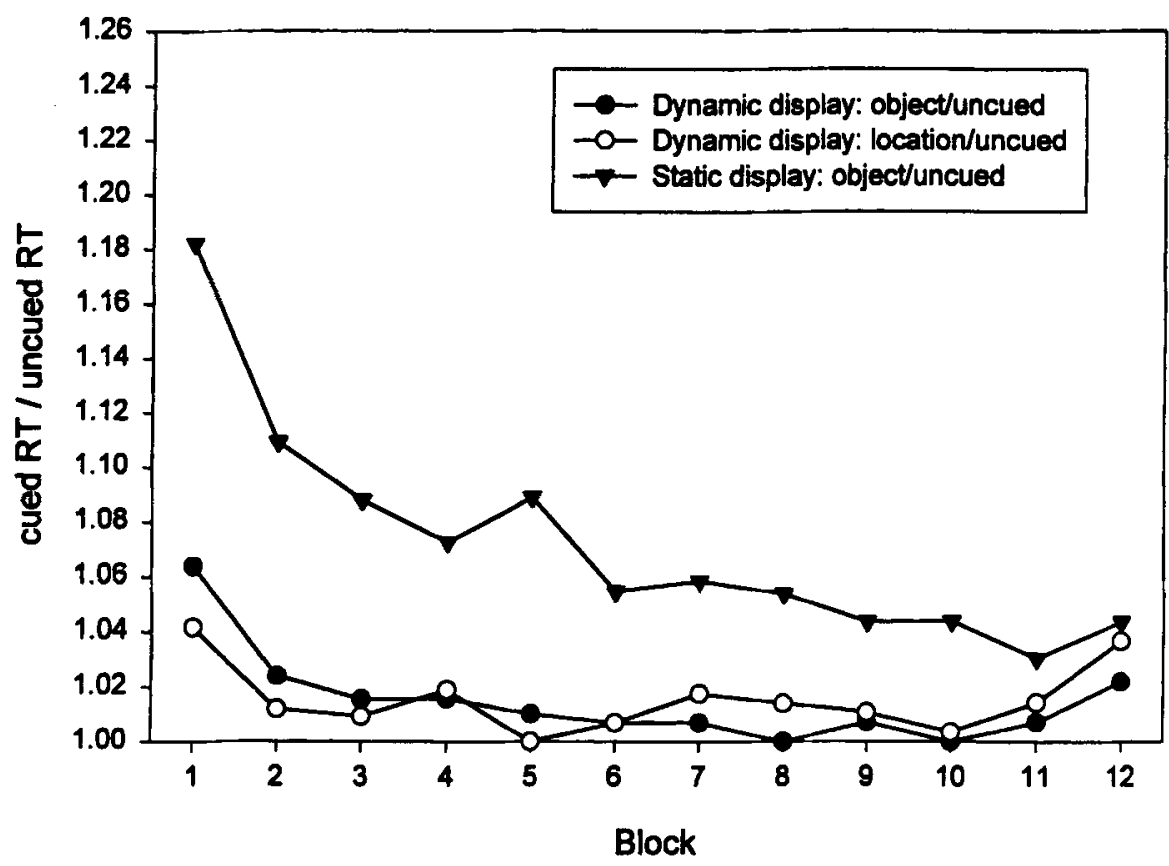

Figure 1. The results from Experiments 1 (dynamic display) and 2 (static display) of Weaver et al. (1998; Tables 2 and 3). The proportions were calculated by dividing the median cued RT by the median uncued RT. Thus, greater proportions represent more inhibition of return. There were four blocks per day of testing. In the static display experiment, there were two uncued conditions (one termed location and the other uncued). These were averaged together in the calculation and termed uncued in the present figure. 
decreased from $23 \mathrm{msec}$ in the first block of 150 trials (90 test and 60 catch trials) to $6 \mathrm{msec}$ in the last block, while the location-based inhibition decreased from $15 \mathrm{msec}$ in the first block to almost $0 \mathrm{msec}$ in the subsequent blocks. Likewise, the magnitude of the object plus location inhibition in the second experiment decreased from $57 \mathrm{msec}$ in the first block to $11 \mathrm{msec}$ in the last block. However, in addition to the decrease in inhibition with practice, there was also a decrease in the overall RTs with practice. Thus, the reduction in inhibition may simply have occurred because of a floor effect on the overall latencies. In order to examine this, we have plotted the inhibition of return effects reported by Weaver et al. (their Tables 2 and 3 ) as proportions by dividing the cued RTs by the uncued RTs for each block. Thus, a score of 1.0 would indicate that the cued RT was equal to the uncued RT, and greater amounts of inhibition of return would be indicated by larger proportions. The proportional data from Weaver et al. are presented in Figure 1.

Figure 1 shows a clear and consistent pattern of the reduction in inhibition of return with practice. All of the decease in the inhibition occurred in the 1 st day of testing, a session that consisted of four blocks of 20 practice trials and 150 test trials each. After the 1 st day of testing, the amount of inhibition did not change with practice but rather stayed at a relatively consistent, low level (although there was a small rebound in the static display experiment at the beginning of the 2 nd day, it lasted only until the fifth block). Weaver et al. (1998) suggested that Müller and von Mühlenen (1996) failed to find objectbased inhibition of return because of the extensive practice sessions they provided to each participant before they began recording data. The proportions presented in Figure 1 certainly bear out this suggestion; Weaver et al. found considerable inhibition of return on the 1 st day of testing, which decreased during the 1 st day with practice, and then very little (if any) in the subsequent days of testing.

The purpose of the present study was twofold. The first purpose was to attempt to replicate the practice effects on inhibition of return observed by Weaver et al. (1998). This is especially important because the results that might arise from an experiment involving inhibition of return may not be due a specific experimental manipulation but to the number of practice trials or experimental trials in the experiment. The second purpose was to examine Weaver et al.'s notion that some of the decrease in the inhibition may be due to habituation. To examine this issue, the cue stimulus and target stimulus, which remained constant from the first trial onward, were switched in the last block. Presumably changing the cue and target stimuli in the last block of trials would eliminate any habituation that had built up over the previous trials and would therefore produce an increase in the inhibitory effect.

\section{EXPERIMENT 1}

In most experiments that examine inhibition of return, participants are required to respond to a moderate num- ber of trials in a single experimental session. To give some typical examples from single-session experiments, Pratt et al. (1997, Experiment 1) used 240 trials, Pratt (1995) used 280 trials, Tipper et al. (1994, Experiment 1) used 384 trials, and Maylor and Hockey (1985) used 480 trials. Weaver et al. (1998) conducted a lengthy four-session experiment with over 1,000 trials in total. In the present experiment, we examined whether the practice effects found by Weaver et al. also might affect the magnitude of inhibition of return in a more typical single-session experiment of approximately 350 trials. Near the end of each session, the stimuli used for the cue and target were switched (i.e., the cue stimulus became the target stimulus and vice versa) so that, if a decrease in inhibition with practice was found, it would be possible to examine the habituation explanation put forward by Weaver et al. In addition, we used smaller blocks than did Weaver et al. (48 test trials per block) in order to allow a finer grained examination of the practice effect.

In addition, the present experiment also examined whether the effect of practice on inhibition of return type would be mediated by the nature of the response. In most experiments that have examined inhibition of return with a detection task, the response was either a simple keypress with catch trials (e.g., Weaver et al., 1998) or a choice keypress based on the location of the target, without catch trials (e.g., Pratt \& Abrams, 1995). Because we were interested in examining the role of practice in a "typical" inhibition of return experiment, both types of responses were used in separate groups.

\section{Method}

Participants. Twenty undergraduate students from Lakehead University participated in the experiment. Each participant volunteered his/her time.

Apparatus and Procedure. Participants were seated at a table approximately $40 \mathrm{~cm}$ directly in front of a computer monitor. A computer keyboard was placed on the table so that the participants could easily reach the keys.

At the start of each trial, a display consisting of a central fixation dot and a peripheral box on either side of the fixation dot was presented. The boxes were centered $5^{\circ}$ from the fixation dot and were $1^{\circ}$ high by $1^{\circ}$ wide. The participants were instructed to remain fixated on the fixation dot throughout the duration of each trial. Following a delay of $1,500 \mathrm{msec}$, a cue was presented in one of the peripheral boxes and remained present for $200 \mathrm{msec}$. Following the removal of the cue, a delay of $900 \mathrm{msec}$ occurred before the target was presented. On cued trials, the target appeared in the same box that had been previously cued. On uncued trials, the target appeared in the box opposite from the cued box. The participants were instructed to respond to the targets as quickly and as accurately as possible. One group of 10 participants, the simple reaction time (SRT) group, was instructed to press the space bar with the dominant hand as soon as the appearance of the target was detected. Another group of 10 participants, the choice reaction time (CRT) group, was instructed to press the " $Z$ " key with the left hand if the target appeared in the left box and the "/" with the right hand if the target appeared in the right box.

Design. The CRT group performed seven blocks of 48 test trials (no catch trials) and the SRT group performed seven blocks of 60 trials (48 test trials and 12 catch trials in which no target was presented). The catch trials were added to the SRT group in order to reduce the number of anticipatory responses. These were not needed 
in the CRT groups due to the spatial uncertainty of the response. For the first six blocks of each group, the cue was a filled-in circle and the target was a filled-in square. However, in the last block for each group, the stimuli were switched so that the cue was the filled-in square and the target was the filled-in dot (called the switch block). Within each block, cued and uncued trials were equally likely, as was the location of the targets (left or right box). In the SRT group, the catch trials were randomly distributed throughout each block. A short rest period was given at the end of each block, and the participants were instructed to begin the next block when they felt ready to do so.

\section{Results and Discussion}

As in all the present experiments, trials in which the RT was less than $100 \mathrm{msec}$ or greater than $800 \mathrm{msec}$ were considered errors and not included in the analysis. The mean RTs from the correct trials from Experiment 1 are shown in Table 1. The mean RTs were analyzed with a 2 (group: SRT or CRT) $\times 2$ (trial type: cued or uncued) $\times 6$ (block) analysis of variance (ANOVA) (the switch block was excluded). Main effects were found for block $[F(5,18)=12.3, p<.0001]$ (RTs decreased with increasing practice $)$ and for trial type $[F(1,18)=86.0, p<.0001]$ (RTs were slower in cued trials than in uncued trials, the typical inhibition of return effect). No main effect was found for group $[F(1,18)<1]$. The block $\times$ group interaction was not significant $[F(5,90)=2.1, p>.05]$, although there was a trend for the CRT group to show a greater reduction in RT with practice. The trial type $\times$ group interaction was also significant, with more inhibition of return (the difference between cued and uncued trials) in the SRT group than in the CRT group $[F(1,18)=$ $11.4, p<.005]$. Importantly, neither the block $\times$ trial type interaction nor the block $\times$ trial type $\times$ group interaction was significant $[F \mathrm{~s}(5,90)<1]$.

In order to further examine the effect of practice on inhibition of return, proportions were calculated by dividing the cued RT by the uncued RT per block for each participant. These data are presented in Figure 2 and were analyzed with a 2 (group) $\times 5$ (block) ANOVA. As in the RT analysis, a main effect was found for group $[F(1,18)=$ $11.7, p<.005]$ (more inhibition in the SRT group), but there was no effect of block $[F(5,90)<1]$, nor was there a

Table 1

Mean RTs (in Milliseconds) for the Correct Responses, the Amount of Inhibition of Return (IOR), and the Percentage of Errors in Experiment 1

\begin{tabular}{|c|c|c|c|c|c|c|c|c|}
\hline \multirow[b]{3}{*}{ Block } & \multicolumn{8}{|c|}{ Group } \\
\hline & \multicolumn{4}{|c|}{ CRT } & \multicolumn{4}{|c|}{ SRT } \\
\hline & Cued & Uncued & IOR & Error & Cued & Uncued & IOR & Error \\
\hline 1 & 351 & 336 & 15 & 1.4 & 350 & 306 & 44 & 1.2 \\
\hline 2 & 326 & 301 & 25 & 1.0 & 344 & 287 & 57 & 1.0 \\
\hline 3 & 325 & 302 & 23 & 1.1 & 342 & 295 & 47 & 0.8 \\
\hline 4 & 314 & 293 & 21 & 1.3 & 340 & 284 & 54 & 1.1 \\
\hline 5 & 320 & 291 & 29 & 1.4 & 334 & 288 & 46 & 1.0 \\
\hline 6 & 311 & 287 & 24 & 1.0 & 331 & 287 & 44 & 0.8 \\
\hline 7 & 310 & 285 & 25 & 1.1 & 330 & 286 & 44 & 1.0 \\
\hline
\end{tabular}

CRT, choice reaction time; SRT, simple reaction time. block $\times$ group interaction $[F(5,90)<1]$. Although there were virtually no differences in the proportion scores between the sixth block and the switch block, planned within-condition $t$ tests were conducted, and these indicated that the switch block did not produce an increase in the inhibition $[t \mathbf{s}(9)<1]$.

The percentage of error trials is also presented in Table 1 and was analyzed with the same 2 (group) $\times 2$ (trial type) $\times 6$ (block) ANOVA. There were no differences in the percentages of errors between any of the conditions, as indicated by a lack of main effects and interaction effects $[F \mathrm{~s}(1,18)<1, F \mathrm{~s}(5,90)<1]$. In general, very few errors were made; no participant produced more than two errors in any given single condition for any given block.

Overall, the results of the present experiment indicate that the magnitude of the inhibition of return effect did not decrease with practice for either the SRT or the CRT group. In the CRT group, there was $15 \mathrm{msec}$ of inhibition in the first block of 48 test trials and $24 \mathrm{msec}$ of inhibition in the second-to-last block (the last block before the switch in the cue and target stimuli). In the SRT group, there was $44 \mathrm{msec}$ of inhibition in the first block and $44 \mathrm{msec}$ in the second-to-last block. Thus, over the space of 288 test trials, there was no evidence of any effect of practice on inhibition of return. The fact that there was no practice-related decrease in the inhibition severely limited the chance of finding a release from habituation with the switch of the cue and target stimuli in the last block. This was indeed the case, since the amount of inhibition found in the last block was almost identical, in both groups, to the amount of inhibition found in the second-to-last block. Despite the lack of changes in inhibition of return, there was strong evidence that the practice did affect performance on the task, since overall RTs for both SRT and CRT groups showed a marked decrease over the course of the experiment.

The discrepancy between the present findings and those of Weaver et al. (1998) is especially apparent in the proportional data (Figures 1 and 2). In fact, one of the advantages of plotting the proportional data is that the results of separate experiments can be directly compared on a standardized measure. On the 1 st day of testing, Weaver et al. found a decreasing monotonic function of the proportion of inhibition, whereas there was no such decrease in the function from the present single-session experiment. Thus, Figures 1 and 2 show clearly that practice did not have the expected effect on inhibition of return in the present experiment.

One interesting difference between the SRT and CRT groups concerns the overall amounts of inhibition found in each group. Across all trials, there was $33 \mathrm{msec}$ of inhibition of return in the CRT group and $48 \mathrm{msec}$ of inhibition in the SRT group, even though the overall RTs (across cued and uncued trials) for each group were almost equal. One possibility is that the catch trials in the SRT group produced a greater overall amount of inhibition. It may be that the slowing associated with inhibition 


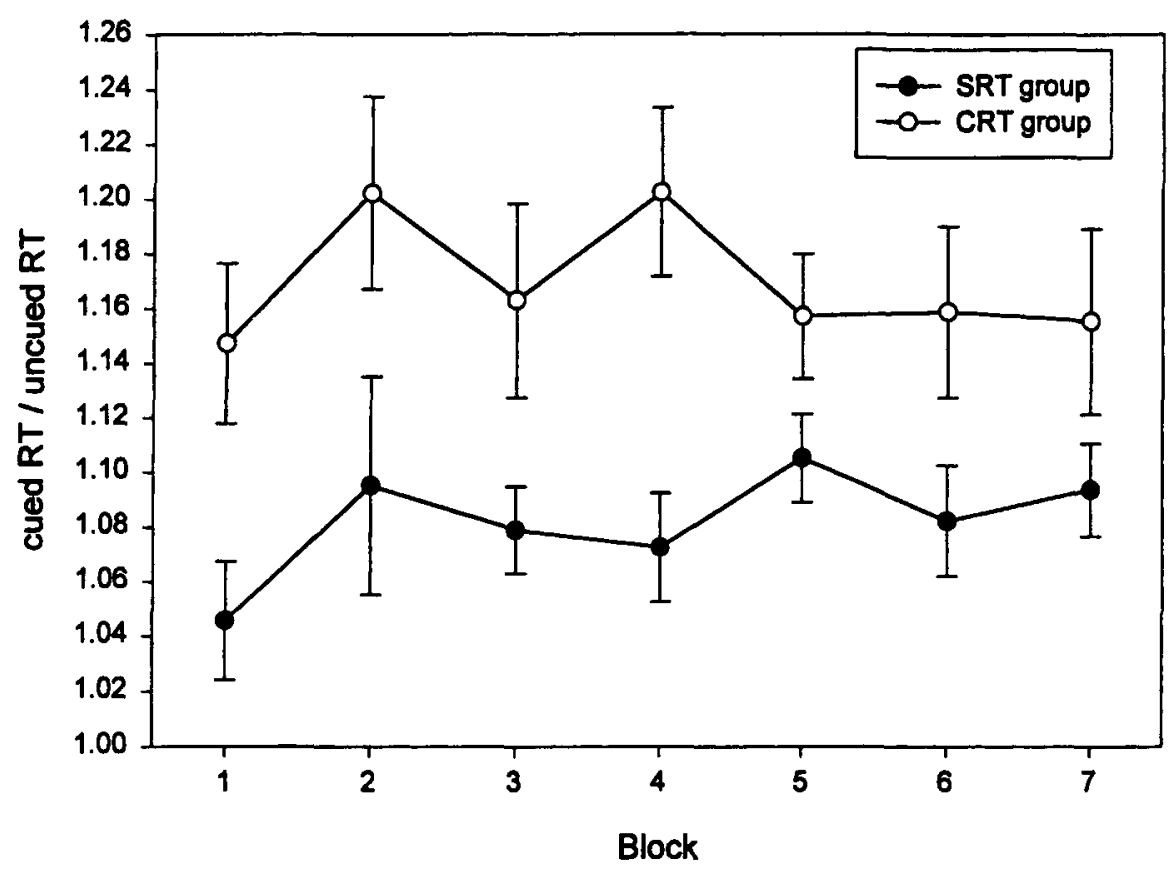

Figure 2. The time course of the proportional effect of inhibition of return (mean cued RT/mean uncued RT) for Experiment 1 . Filled circles represent the simple reaction time (SRT) group, open circles the choice reaction time (CRT) group. Errors bars are standard errors.

of return combines with a general inhibitory "set" in which participants are prepared to inhibit the production of a response (i.e., catch trials). The suggestion that the slower responses to targets at cued locations may be partially due to inhibited motor responses is consistent with the notion that inhibition of return may be best described as a phenomenon of the motor system and not the attentional system (Klein \& Taylor, 1994). It should be noted, however, that there is also evidence that the locus of inhibition of return is the attentional system and not necessarily the motor system (Pratt et al., 1997). Thus, it seems possible that the mechanisms that produce slower responses to cued locations may reside in both systems and that these mechanisms combine to produce the overall inhibitory effect.

Overall, the findings from the present experiment were somewhat unexpected because we failed to replicate the effects of practice on inhibition of return reported by Weaver et al. (1998). There were, however, some important differences between the Weaver et al. experiment and present experiment. First, Weaver et al. used a display consisting of three peripheral boxes, whereas the present experiment used a display consisting of two peripheral boxes. Second, and probably more importantly, Weaver et al. had participants perform over 1,000 test trials, whereas the present experiment had participants perform fewer than 400 test trials. These issues were addressed in a second experiment.

\section{EXPERIMENT 2}

The second experiment was designed to fulfill the twofold purpose of the first experiment: to replicate the Weaver et al. (1998) finding of decreased inhibition with practice and to test the notion that habituation was partly responsible for that decrease. We made two major changes in the present experiment. First, the number of trials was increased to make the total number of trials more similar to the number used by Weaver et al. Second, one group of participants used the two-box display used in the first experiment whereas another group of participants used a three-box display similar to that of Weaver et al. In this manner, the effect of stimulus display on the change in the inhibition over trials could be determined. As in Weaver et al., the experiment used simple keypress responses and catch trials.

\section{Method}

Participants. Twenty undergraduate students from Lakehead University participated in the experiment. Each participant volunteered his/her time.

Apparatus and Procedure. The apparatus was exactly the same as that used in Experiment 1. The procedure for the 10 participants in the two-box group was exactly the same as the procedure used by the SRT group except that instead of the seven blocks used in Experiment 1, the two-box group in the present experiment performed 19 blocks ( 18 test blocks and 1 switch block). The procedure for the 10 participants in the three-box group was the same as that in 
the two-box group, but the display was different. The display in the three-box group consisted of three boxes, each centered $5^{\circ}$ from the fixation point and spaced equidistantly from each other (similar to the display used by Weaver et al., 1998). Thus, the three boxes were located on a imaginary circle with $120^{\circ}$ of angle between each box.

Design. Each group performed 60 trials per block (48 test trials and 12 catch trials). As in the previous experiment, the cue was a filled-in circle and the target was a filled-in square for the first 18 blocks. In the last block, the stimuli were switched so that the cue was the filled-in square and the target was the filled-in dot. Within each block, cued and uncued trials were equally likely, as was the location of the targets, and the catch trials were randomly distributed throughout each block. Thus, in the two-box group, there were 24 cued and 24 uncued trials in each block, whereas in the three-box group, there were 16 cued and 32 uncued trials in each block. Also as before, rest breaks were provided between each block.

\section{Results and Discussion}

The mean RTs from the correct trials from Experiment 2 are shown in Table 2 . The mean RTs were analyzed with a 2 (group: two-box or three-box) $\times 2$ (trial type: cued or uncued) $\times 18$ (block) ANOVA (the switch block was not included). Main effects were found for block $[F(1,17)=$ $7.6, p<.0001]$ (RTs decreased with increasing practice) and for trial type $[F(1,18)=102.8, p<.0001]$ (RTs were slower in cued trials than in uncued trials, indicating the presence of inhibition of return). There was also a main effect of group $[F(1,18)=4.2, p<.05]$ (RTs were faster in the two-box group than in the three-box group). The trial type $\times$ group interaction was significant $[F(1,18)=$ $7.6, p<.02$ ], with more inhibition of return in the twobox group than in the three-box group. The block $\times$ group interaction was not significant $[F(1,18)<1.3, p>.20]$.

Table 2

Mean RTs (in Milliseconds) for the Correct Responses, the Amount of Inhibition of Return (IOR), and the Percentage of Errors (Averaged Over Cued and Uncued Trials) in Experiment 2

\begin{tabular}{|c|c|c|c|c|c|c|c|c|}
\hline \multirow[b]{3}{*}{ Block } & \multicolumn{8}{|c|}{ Group } \\
\hline & \multicolumn{4}{|c|}{ Two-Box } & \multicolumn{4}{|c|}{ Three-Box } \\
\hline & Cued & Uncued & IOR & Error & Cued & Uncued & IOR & Error \\
\hline 1 & 365 & 312 & 53 & 1.5 & 387 & 363 & 24 & 2.1 \\
\hline 2 & 348 & 295 & 53 & 0.4 & 350 & 331 & 19 & 1.3 \\
\hline 3 & 347 & 289 & 58 & 1.0 & 350 & 325 & 25 & 0.4 \\
\hline 4 & 335 & 288 & 47 & 0.8 & 354 & 326 & 28 & 3.1 \\
\hline 5 & 328 & 290 & 38 & 1.7 & 347 & 319 & 28 & 1.9 \\
\hline 6 & 334 & 289 & 45 & 1.5 & 365 & 327 & 38 & 1.9 \\
\hline 7 & 319 & 277 & 42 & 0.8 & 359 & 328 & 31 & 2.3 \\
\hline 8 & 325 & 285 & 37 & 1.7 & 355 & 326 & 29 & 2.7 \\
\hline 9 & 321 & 287 & 34 & 1.0 & 353 & 331 & 22 & 1.9 \\
\hline 10 & 328 & 279 & 39 & 1.9 & 352 & 334 & 18 & 1.3 \\
\hline 11 & 323 & 277 & 46 & 1.0 & 346 & 326 & 20 & 2.3 \\
\hline 12 & 329 & 281 & 48 & 2.7 & 344 & 322 & 22 & 1.9 \\
\hline 13 & 324 & 286 & 38 & 1.9 & 348 & 326 & 22 & 1.3 \\
\hline 14 & 328 & 288 & 40 & 0.8 & 333 & 315 & 18 & 2.1 \\
\hline 15 & 318 & 282 & 36 & 1.3 & 339 & 316 & 23 & 1.9 \\
\hline 16 & 323 & 280 & 43 & 2.5 & 349 & 315 & 34 & 1.5 \\
\hline 17 & 323 & 288 & 35 & 2.3 & 345 & 322 & 23 & 0.6 \\
\hline 18 & 326 & 286 & 40 & 1.9 & 345 & 323 & 23 & 1.9 \\
\hline 19 & 316 & 271 & 45 & 2.3 & 356 & 323 & 33 & 2.1 \\
\hline
\end{tabular}

Once again, block did not interact with either trial type or trial type and group $\left[F_{\mathrm{s}}(17,306)<1\right]$.

As in the first experiment, proportions were calculated by dividing the cued RT by the uncued RT for each block. These data are presented in Figure 3 and were analyzed with a 2 (group) $\times 18$ (block) ANOVA. As in the $\mathrm{RT}$ analysis, a main effect was found for group $[F(1,18)=$ $9.8, p<.01]$ (more inhibition in the two-box group), but there was no effect of block $[F(17,306)<1]$ nor was there a block $\times$ group interaction $[F(17,306)<1]$. Planned within group $t$ tests were conducted on the proportions between the 18th block and the switch block, and these indicated that there was no increase in inhibition at the switch block for either group [ $t \mathrm{~s}(9)<1.8, p \mathrm{~s}>.10]$.

The percentage of error trials in the present experiment is also presented in Table 2 and was analyzed with the same 2 (group) $\times 2$ (trial type) $\times 18$ (block) ANOVA. There were no differences in the percentages of errors between any of the conditions, as indicated by a lack of main effects and interaction effects $[F \mathrm{~s}(1,17)<2.0, p \mathrm{~s}>$ $.15 ; F \mathrm{~s}(17,306)<1]$. As in the first experiment, very few errors were made; no participant produced more than three errors in any given condition in any given block.

The results from the present experiment are very similar to the results of the first experiment. As found earlier, the overall RT decreased with practice. More importantly, there was also no evidence for decreases in the magnitude of inhibition of return with practice. The threebox group produced $25 \mathrm{msec}$ of inhibition in the first block and $22 \mathrm{msec}$ of inhibition in the second-to-last block (the last block before the switch of the cue and target stimuli). Although the inhibition in the two-box group decreased from $53 \mathrm{msec}$ in the first block to $40 \mathrm{msec}$ in the second-to-last block, the greatest amount of inhibition was found in the third block, and the size of the effect averaged about $40 \mathrm{msec}$ from the fourth block to the secondto-last block. This can also be seen by comparing the proportional data presented in Figures 1 and 3. It is worth emphasizing no practice effects were found even though participants performed almost 1,000 test trials (over 1,000 trials including catch trials) in a single session.

Although no practice effects were found in the present experiment, more inhibition of return was found in the two-box group than in the three-box group. This may be because it is more difficult to inhibit one of three locations than it is to inhibit one of two locations. More specifically, it is possible that there may be some cognitive load associated with keeping track of locations over the duration of the inhibitory effect (i.e., about 2,000 msec). Presumably this load increases as the number of possible target locations increases, and this results in a decrease in the inhibitory effect. However, the role of the number of possible target locations on inhibition of return has not been examined in depth. Wright and Richard (1993) found equal magnitudes of inhibition while varying the number of simultaneously cued locations, but the present results 


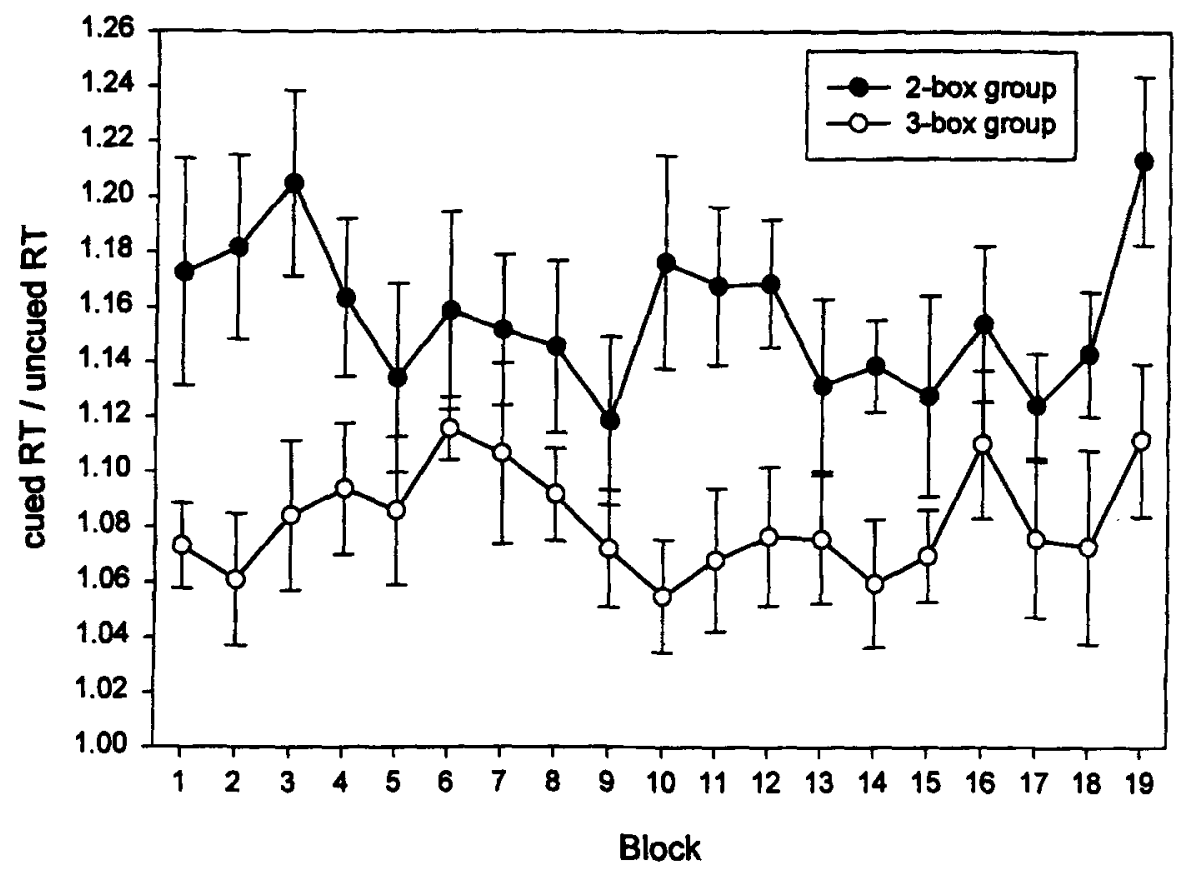

Figure 3. The time course of the proportional effect of inhibition of return (mean cued RT/mean uncued RT) for Experiment 2. Filled circles represent the two-box group, open circles the three-box group. Errors bars are standard errors.

suggest that the number of potential target locations may have a significant impact on the magnitude of the inhibitory effect.

Overall, the results from the first two experiments revealed no practice-related changes in the amount of inhibition of return. However, there were some differences in the visual displays used by Weaver et al. (1998) and those used in the present two experiments. To account for the possible role of these differences, a more rigorous replication of the Weaver et al. procedure was conducted in Experiment 3.

\section{EXPERIMENT 3}

The present experiment was conducted in order to replicate the second experiment of Weaver et al. (1998). We chose to replicate their second experiment for two reasons. First, the static display they used produced much larger inhibition of return effects than did their dynamic display, which may have yielded a better chance of observing changes in the inhibition of return (i.e., less chance of finding floor effects). Second, their static display experiment was more comparable to Experiments 1 and 2 of the present study, in which static displays were also used.

\section{Method}

Participants. Sixteen undergraduate students from the University of Toronto participated in the experiment. All received course credit for their participation.

Apparatus and Procedure. The apparatus was exactly the same as that used in Experiment 1. The procedure for this experiment was very similar to that used by Weaver et al. (1998) in their second experiment. The display consisted of a central fixation box (dark gray) surrounded by three peripheral target boxes (blue, red, and magenta, respectively), and these were presented on a light-gray background. Each subtended $1^{\circ}$ of visual angle, and the peripheral boxes were spaced equidistantly $\left(120^{\circ}\right.$ apart) on an imaginary circle with a radius of $5.5^{\circ}$ from the center of the fixation box point. The display was presented for $1,100 \mathrm{msec}$ and then on the peripheral boxes was replaced by a cue. The cue consisted of enlarging the peripheral box to $1.5^{\circ}$, filling in the box with a white box subtending $1^{\circ}$, and filling in the white box with a colored box subtending $0.5^{\circ}$. This cue was presented for $100 \mathrm{msec}$ and then replaced by the original display. Two hundred msec later, the central fixation box was cued in exactly the same manner for $100 \mathrm{msec}$ and was replaced by the original display. Following a delay of $450 \mathrm{msec}$, a target appeared in one of the peripheral boxes on $60 \%$ of the trials. The target consisted of white box (subtending $1^{\circ}$ ) superimposed on one of the colored boxes. Participants were instructed to withhold making responses if no target was presented (catch trial) and to press the space bar as quickly and accurately as possible if a target was presented. A short tone was sounded on error trials.

Design. Each participant performed 75 trials per block, which consisted of 45 test trials and 30 catch trials. A total of eight blocks were performed by each participant, for a total of 600 trials. Within each block, the cue was equally likely to appear at any of the three peripheral boxes, as was the target. The starting position of the three boxes was randomized on each trial, although the boxes were always $120^{\circ}$ apart. Once again, rest breaks were provided after each block.

The number of trials in the present experiment was chosen for two reasons. First, the decrease in inhibition found by Weaver et al. (1998) occurred almost entirely within their first session of practice, which consisted of 600 trials. Second, most inhibition of return experiments occur within a single session and contain fewer than 600 trials. Also, we did not include practice trials in the present experiment, whereas Weaver et al. provided 20 practice trials every 
Table 3

Mean RTs (in Milliseconds) for the Correct Responses, the Amount of Inhibition of Return (IOR), and the Percentage of Errors in Experiment 3

\begin{tabular}{ccccccccc}
\hline & \multicolumn{4}{c}{ Mean RTs } & & \multicolumn{3}{c}{ Mean Median RTs } \\
\cline { 2 - 4 } Block & Cued & Uncued & IOR & Error & & Cued & Uncued & IOR \\
\hline 1 & 419 & 381 & 38 & 1.1 & & 415 & 372 & 43 \\
2 & 412 & 379 & 33 & 0.8 & & 418 & 379 & 39 \\
3 & 403 & 374 & 29 & 0.8 & & 408 & 369 & 39 \\
4 & 399 & 368 & 31 & 1.2 & & 392 & 361 & 31 \\
5 & 393 & 356 & 37 & 0.5 & & 383 & 343 & 40 \\
6 & 380 & 356 & 24 & 0.7 & & 375 & 343 & 32 \\
7 & 387 & 359 & 28 & 1.4 & & 383 & 347 & 36 \\
8 & 379 & 351 & 28 & 1.5 & 376 & 341 & 35 \\
\hline
\end{tabular}

150 trials. We did not include the practice trials because we wanted to examine the effects of practice across all trials and did not want to exclude any data. Also, we used blocks of 75 trials, whereas Weaver et al. used blocks of 150 trials. This was done to provide a finer detail of analysis of the practice effects than was possible in the Weaver et al. study.

\section{Results and Discussion}

The mean RTs from the correct trials from Experiment 3 are shown in Table 3 . The mean RTs were analyzed with a 2 (trial type: cued or uncued) $\times 7$ (block) ANOVA (the switch block was excluded). Main effects were found for block $[F(6,90)=3.9, p<.002]$ (RTs decreased with increasing practice) and for trial type $[F(1,15)=111.8$, $p<.0001]$ (RTs were slower in cued trials than in uncued trials, indicating the presence of inhibition of return).
Unlike the results from Weaver et al. (1998), and consistent with the findings from the previous experiments, no trial type $\times$ block interaction was found $[F \mathrm{~s}(6,90)<1]$. The lack of a practice effect was confirmed with a oneway ANOVA (block) done on the proportional data (cued RT divided by uncued RT per block) $[F(6,90)<1]$.

The proportional data derived from the mean RT scores appear in Figure 4 with the proportional data derived from the median RT scores (see below). Once again, a planned $t$ test on the proportional data in the second-to-last block and switch block was not significant $[t(15)<1]$.

The percentage of error trials in the present experiment is also presented in Table 3 and was analyzed with the same 2 (trial type) $\times 8$ (block) ANOVA. There were no differences in the percentages of errors between any of the conditions, as indicated by a lack of main effects and interaction effects $\left[F_{\mathrm{s}}(1,18)<2.0, p \mathrm{~s}>.15 ; F_{\mathrm{s}}(6,108)<1\right]$. As in the first experiment, very few errors were made; no participant produced more than two errors in any given condition in any given block.

One important difference between the present experiments and the Weaver et al. (1998) experiments is that we examined mean RT values, whereas Weaver et al. examined mean median RTs. Therefore, a 2 (trial type) $\times 7$ (block) ANOVA was conducted on the mean median RTs from each participant. These values also appear in Table 3. Similar to the mean RT results, main effects were found for trial type $[F(1,15)=126, p<.0001]$ and for block $[F(6,90)=4.9, p<.0005]$. Also similar to the mean RT analysis, no interaction was found between trial type and

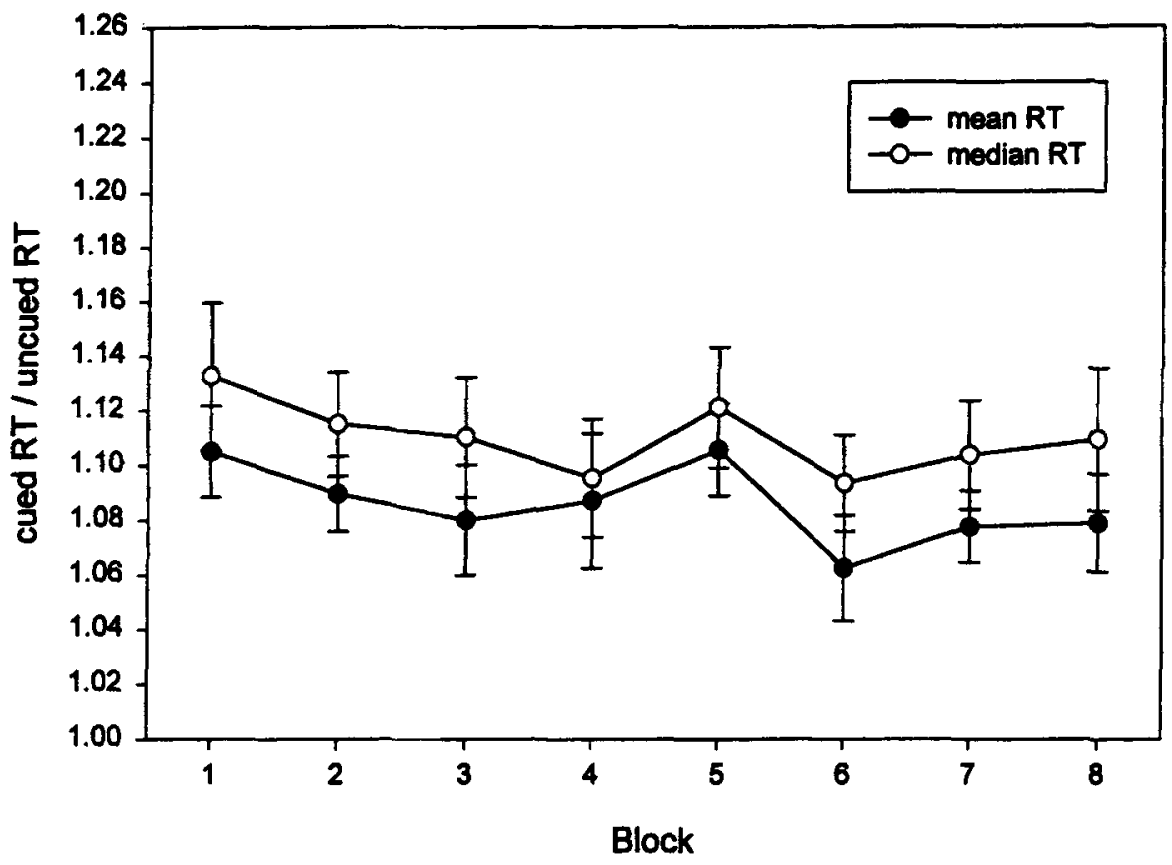

Figure 4. The time course of the proportional effect of inhibition of return (cued RT/uncued RT) for Experiment 3. Filled circles represent the proportions calculated from the mean data, open circles from the median data. Errors bars are standard errors. 
block $[F(6,90)<1]$, indicating that the amount of inhibition did not change with practice. As before, the lack of a practice effect was confirmed with a one-way ANOVA (block) done on the proportional data (median cued RT divided by median uncued RT per block) $[F(6,90)<1]$ (Figure 4).

The results from the present experiment replicate the results from the previous experiments in this study and do not replicate the Weaver et al. (1998) finding that inhibition of return was diminished with practice. No practice effects occurred even though the procedure used in the present experiment was very similar to that used by Weaver et al. in their second experiment, in which they found that the amount of inhibition of return decreased from $57 \mathrm{msec}$ in the first block to $20 \mathrm{msec}$ in the fourth block. Over the same number of trials ( 600 total trials, 360 test trials, and 240 catch trials), we found that the amount of inhibition decreased from 32 to $20 \mathrm{msec}$. However, this decrease was not monotonic, with the most inhibition being found in the fourth block. In fact, an examination of the individual data indicated that only 2 of the 16 participants showed a monotonic decrease in inhibition like that found over the first four blocks for the median RTs of Weaver et al. The lack of a practice effect similar to that reported by Weaver et al. in the present experiment, for mean or median RT scores, can be further confirmed by comparing Figures 1 and 4.

\section{GENERAL DISCUSSION}

The present three experiments were designed to examine the role of practice effects on the magnitude of inhibition of return. Using typical inhibition of return paradigms, five groups of participants received large numbers of trials in which they detected targets at cued or uncued locations. Robust inhibition of return effects were found in all five groups. However, no decrease in the magnitudes of the inhibitory effects were found for any of the groups across the experimental sessions, even though the overall RTs did show marked decreases with practice. Using proportional analyses (cued RT divided by uncued RT), we confirmed that the inhibition of return effect did not decrease with the overall reduction in latencies that occurred with practice.

The failure to find practice effects on inhibition of return in the present study was unexpected. Whereas Weaver et al. (1998) found robust decreases with practice for object- and location-based inhibition (using dynamic displays), as well as for the combination of the two (using static displays), there was no evidence of such changes in the present study, which used static displays to examine the combination of object- and location-based inhibition. The findings of the present experiments suggest that the practice-related changes reported by Weaver et al. may not be that common and may not generalize to other inhibition of return paradigms. This is especially impor- tant because the majority of studies on inhibition of return use single-session experiments with fewer than 600 trials per participant.

It is not immediately clear why Weaver et al. (1998) were able to find robust practice effects with inhibition of return, whereas in the present three experiments, we were not. The only difference of note in the methods between the two studies was that the participants in Weaver et al. were required to press a key to initiate each trial, whereas trials in the present experiments began automatically after a fixed intertrial interval. However, it is unlikely that the present results were due to increasing fatigue in the participants, because they were given rest breaks at relatively short intervals (between 48 and 75 trials). It also seems unlikely that such a small and incidental difference in experimental design would lead to the difference in results.

Overall, the major conclusion that may be drawn from the present experiments is that decreases in inhibition of return with practice are unlikely to occur in a singlesession experiment using static displays and containing between 300 and 1,000 trials. Thus, the present findings indicate that investigators using such inhibition of return paradigms may be fairly secure in knowing that the inhibitory effect is unlikely to vary, to any noticeable extent, from the start of an experiment to the end of an experiment. This is important because the vast majority of studies that use inhibition of return to examine the inhibitory functioning of the attentional system make use of paradigms with these parameters. However, if expected inhibition of return effects are not found in an experiment, the Weaver et al. (1998) findings do suggest that one factor to examine would be possible decreases in the size of the inhibitory effect over the course of the experiment. This may be especially important in experiments that use dynamic displays, as suggested by the results of Müller and von Mühlenen (1996) and Weaver et al. (1998).

\section{REFERENCES}

Abrams, R. A., \& DobKin, R. S. (1994). Inhibition of return: Effects of attentional cueing on eye movement latencies. Journal of Experimental Psychology: Human Perception \& Performance, 20, 467-477.

KLEIN, R. M. (1988). Inhibitory tagging system facilitates visual search. Nature, 334, 430-431.

Klein, R. M., \& TAYloR, T. L. (1994). Categories of cognitive inhibition with reference to attention. In D. Dagenbach \& T. H. Carr (Eds.), Inhibitory processes in attention, memory, and language (pp. 113150). San Diego: Academic Press.

LABerge, D., \& SAMuels, S. J. (1974). Toward a theory of automatic information processing in reading. Cognitive Psychology, 6, 293-323.

Lupiáñez, J., Milán, E. G., Tornay, F. J., Madrid, E., \& Tudela, P. (1997). Does IOR occur in discrimination tasks? Yes, it does, but later. Perception \& Psychophysics, 59, 1241-1254.

Maylor, E. (1985). Facilitatory and inhibitory components of orienting in visual space. In M. 1. Posner \& O. S. M. Marin (Eds.), Attention and performance XI (pp. 189-204). Hillsdale, NJ: Erlbaum.

MAYLOR, E., \& HoCKEY, R. (1985). Inhibitory component of externally controlled covert orienting in visual space. Journal of Experimental Psychology: Human Perception \& Performance, 11, 777-787. 
Mowbray, G. H., \& Rhoades, M. V. (1959). On the reduction of choice reaction times with practice. Quarterly Journal of Experimental Psychology, 11, 16-23.

MǘleR, H. J., \& von Mühlenen, A. (1996). Attentional tracking and inhibition of return in dynamic displays. Perception \& Psychophysics, 58, 224-249.

Posner, M. I., \& CoHEN, Y. (1984). Components of visual orienting. In H. Bouma \& D. G. Bouwhuis (Eds.), Attention and performance $X$ : Control of language processes (pp. 531-556). Hillsdale, NJ: Erlbaum.

PRATT, J. (1995). Inhibition of return in a discrimination task. Psychonomic Bulletin \& Review, 2, 117-120.

PRATT, J., \& ABRAMS, R. A. (1995). Inhibition of return to successively cued spatial locations. Journal of Experimental Psychology: Human Perception \& Performance, 21, 1343-1353.

Pratt, J., \& ABrams, R. A. (1996). Practice and component submovements: The role of feedback in rapid aimed limb movements. Journal of Motor Behavior, 28, 149-156.

Pratt, J., \& ABrams, R. A. (1999). Inhibition of return in discrimination tasks. Journal of Experimental Psychology: Human Perception \& Performance, 25, 229-242.

Pratt, J., Kingstone, A., \& KhOE, W. (1997). Inhibition of return in location- and identity-based choice decision tasks. Perception \& Psychophysics, 59, 964-971.

Proteau, L., Blandin, Y., Alain, C., \& Dorian, A. (1994). The ef- fects of the amount and variability of practice on the learning of a multi-segment motor task. Acta Psychologica, 85, 61-74.

Terry, K. M., VALDES, L. A., \& NeILL, W. T. (1994). Does "inhibition of return" occur in discrimination tasks? Perception \& Psychophysics, $55,279-286$.

TipPer, S. P., Driver, J., \& Weaver, B. (1991). Object-centered inhibition of return of visual attention. Quarterly Journal of Experimental Psychology, 43A, 289-298.

Tipper, S. P., Weaver, B., Jerreat, L. M., \& Burak, A. L. (1994). Object- and environment-based inhibition of return of visual attention. Journal of Experimental Psychology: Human Perception \& Performance, 20, 478-499.

WEAVER, B., LuPí́ÑEZ, J., \& WATSON, F. L. (1998). The effects of practice on object-based, location-based, and static-display inhibition of return. Perception \& Psychophysics, 60, 993-1003.

Wolfe, J. M., \& PokoRNy, C. W. (1990). Inhibitory tagging in visual search: A failure to replicate. Perception \& Psychophysics, 48, 357-362.

WRIGHT, R., \& RICHARD, C. (1993). Inhibition-of-return at multiple locations in visual space. Canadian Journal of Experimental Psychology, 50, 324-327.

(Manuscript received January 9, 1998; revision accepted for publication April 28, 1998.) 